\title{
Hydrodynamics and global embeddings of Taub-NUT spacetime
}

\author{
Soon-Tae Hong* and Sung-Won Kin丹 \\ Department of Science Education, Ewha Womans University, Seoul 120-750 Korea
}

(Dated: December 3, 2018)

\begin{abstract}
On Taub-NUT spacetime, we investigate hydrodynamic properties of perfect fluid spiraling inward toward the spacetime along a conical surface. On the equatorial plane of the Taub-NUT spacetime, we derive radial equations of motion with effective potentials and the Euler equation for steady state axisymmetric fluid. Higher dimensional global embeddings are constructed inside and outside the event horizons of the Taub-NUT spacetime. We also study the effective potentials of particles on the Taub-NUT spacetime in terms of gravitational magnetic monopole strength of the source, total energy and angular momentum per unit rest mass of the particle.
\end{abstract}

PACS numbers: 02.40.Ma, 04.20.Dw, 04.20.Jb, 04.70.Bw, 95.30.L

Keywords: Taub-NUT spacetime, hydrodynamics, Euler equation, global flat embedding

\section{INTRODUCTION}

For many decades, four dimensional solutions of the Einstein equations have been extensively investigated in gravity community. Recently solutions of the Einstein equations or the coupled Einstein-matter equations in higher dimensions have attracted many physical interests since the discovery of supergravity and superstring theory. TaubNUT metric [1] is a local analytic solution of the vacuum Einstein equations. When the metric is expressed in Schwarzschild-like coordinates, we have coordinate singularity occurring at certain values of the radial coordinate where $g_{r r}$ becomes infinite and corresponds to bifurcate Killing horizons. The Taub-NUT spacetime is involved in many modern studies in general relativity. Hawking has proposed that the Euclidean Taub-NUT metric could give rise to the gravitational analogue of the Yang-Mills instanton [2]. In this case the Einstein equations are fulfilled with zero cosmological constant and the manifold is $\mathbf{R}^{4}$ with a boundary which is a twisted three-sphere possessing a distorted metric. The Kaluza-Klein monopole has been obtained by embedding the Taub-NUT gravitational instanton into five dimensional Kaluza-Klein theory [3, 4]. The non-Abelian target space duals of the Taub-NUT spacetime have been also studied in terms of the local isometry group $\mathrm{SU}(2) \times \mathrm{U}(1)$ [5]. The Taub-NUT spacetime has been shown to be related with $\mathrm{SU}(2)$ calons through T-duality [6]. Carter has shown that the Hamilton-Jacobi equation for the geodesics in the Taub-NUT metric separates in certain coordinate systems 77. The gravitomagnetic monopole source effects have been also studied in the Taub-NUT spacetime [8]. In the Kerr-Taub-NUT-de Sitter metrics, separability of the Hamiltonian-Jacobi equation has been studied in higher dimensions [9]. Recently, a rotating Schwarzschild black hole has been studied to investigate effective potentials for null and timelike geodesics of particles and hydrodynamics associated with general relativistic Euler equation for the steady state axisymmetric fluid [10].

A familiar feature of exact solutions to the field equations of general relativity is the presence of singularities. As novel ways of removing the coordinate singularities, the higher dimensional global flat embeddings of the black hole solutions are subjects of great interest both to mathematicians and to physicists. It has been well-known in differential geometry that four dimensional Schwarzschild metric [11] is not embedded in $\mathbf{R}^{5}[12$. Recently, $(5+1)$ dimensional global embedding Minkowski space (GEMS) structure for the Schwarzschild black hole has been obtained [13] to investigate a thermal Hawking effect on a curved manifold [14] associated with an Unruh effect [15] in these higher dimensional spacetime. In $(3+1)$ dimensions, the global flat embeddings inside and outside of event horizons of Schwarzschild and Reissner-Nordström black holes, have been constructed and on these overall patches of the curved manifolds four accelerations and Hawking temperatures have been evaluated by introducing relevant Killing vectors [16]. Recently, the GEMS scheme has been applied to stationary motions in spherically symmetric spacetime [17], and the Banados-Teitelboim-Zanelli black hole [18] has been embedded in (3+2) dimensions to investigate the $\mathrm{SO}(3,2)$ global and $\mathrm{Sp}(2)$ local symmetries [19].

In this paper, exploiting the fact that the coordinates $t$ and $\phi$ are cyclic in the Taub-NUT spacetime we find the timelike Killing field and the axial Killing field, to which we can obtain the conserved energy and the angular momentum per unit rest mass for geodesics. In the Taub-NUT spacetime we then investigate hydrodynamic properties

*Electronic address: soonhong@ewha.ac.kr

${ }^{\dagger}$ Electronic address: sungwon@ewha.ac.kr 
of the perfect fluid spiraling inward toward the Taub-NUT spacetime along a conical surface. On the equatorial plane of the Taub-NUT spacetime, we derive the radial equations of motion with the effective potential. We also study the effective potentials of particles on the Taub-NUT manifold in terms of gravitational magnetic monopole strength of the source, total energy and angular momentum per unit rest mass of the particle. Finally, we construct the higher dimensional global embeddings inside and outside the event horizons of the Taub-NUT manifold.

This paper is organized as follows. In section II we introduce the Taub-NUT spacetime to study its hydrodynamics, and in section III we construct the global flat embeddings inside and outside of event horizon of the Taub-NUT spacetime. Section IV includes summary and discussions.

\section{HYDRODYNAMICS OF TAUB-NUT SPACETIME}

We start with the Taub-NUT 4-metric given by

$$
d s^{2}=-\frac{\Delta}{\Sigma}(d t+2 l \cos \theta d \phi)^{2}+\frac{\Sigma}{\Delta} d r^{2}+\Sigma\left(d \theta^{2}+\sin ^{2} \theta d \phi^{2}\right)
$$

where, in the units $G=c=1$,

$$
\Delta=r^{2}-2 M r-l^{2}, \quad \Sigma=r^{2}+l^{2},
$$

with the parameters $M$ and $l$ being associated with the mass and gravitational magnetic monopole strength of the source. Defining $r_{ \pm}$as

$$
r_{ \pm}=M \pm\left(M^{2}+l^{2}\right)^{1 / 2}
$$

we can rewrite $\Delta$ as

$$
\Delta=\left(r-r_{+}\right)\left(r-r_{-}\right)
$$

Here note that $r_{+}>l$ is an event horizon but $r_{-}$is not since $r_{-}$is negative.

The four velocity is given by

$$
u^{a}=\frac{d x^{a}}{d \tau}
$$

where we can choose $\tau$ to be the proper time (affine parameter) for timelike (null) geodesics. From the equation of motion of a test particle in the Taub-NUT spacetime, the particle initially at rest at infinity spiral inward toward the spacetime along a conical surface of constant $\theta=\theta_{\infty}$ where $\theta_{\infty}$ is the polar angle at infinity. For a fluid which is at rest at infinity and approaches supersonically to the spacetime, one may take the approximation to simplify the hydrodynamical equations

$$
u^{\theta}=\frac{d \theta}{d \tau} \approx 0
$$

As in the Schwarzschild black hole since the coordinates $t$ and $\phi$ are cyclic we have the timelike Killing field $\xi^{a}$ and the axial Killing field $\psi^{a}$. Corresponding to the Killing fields $\xi^{a}$ and $\psi^{a}$ we can then find the conserved energy $E$ and the angular momentum $L$ per unit rest mass for geodesics given as follows

$$
\begin{aligned}
E & =-g_{a b} \xi^{a} u^{b} \\
& =\frac{\left(r-r_{+}\right)\left(r-r_{-}\right)}{r^{2}+l^{2}}\left(u^{t}+2 l u^{\phi}\right), \\
L & =g_{a b} \psi^{a} u^{b} \\
& =-\frac{2 l\left(r-r_{+}\right)\left(r-r_{-}\right)}{r^{2}+l^{2}} u^{t}+\left(\left(r^{2}+l^{2}\right) \sin ^{2} \theta-\frac{4 l^{2}\left(r-r_{+}\right)\left(r-r_{-}\right)}{r^{2}+l^{2}} \cos ^{2} \theta\right) u^{\phi},
\end{aligned}
$$

where $u^{a}$ are four velocity of the locally nonrotating observers defined by (2.5). Moreover, we can introduce a new conserved parameter $\kappa$ defined as

$$
\kappa=-g_{a b} u^{a} u^{b}
$$


whose values are given by $\kappa=1$ for timelike geodesics and $\kappa=0$ for null geodesics.

In the case of geodesics on the equatorial plane $\theta=\pi / 2, u^{t}$ and $u^{\phi}$ are given in terms of $E$ and $L$ as follows

$$
\begin{aligned}
u^{t} & =\frac{r^{2}+l^{2}}{\left(r^{2}+l^{2}\right)^{2}+4 l^{2}\left(r-r_{+}\right)\left(r-r_{-}\right)}\left(\frac{\left(r^{2}+l^{2}\right)^{2}}{\left(r-r_{+}\right)\left(r-r_{-}\right)} E-2 l L\right) \\
u^{\phi} & =\frac{r^{2}+l^{2}}{\left(r^{2}+l^{2}\right)^{2}+4 l^{2}\left(r-r_{+}\right)\left(r-r_{-}\right)}(L+2 l E),
\end{aligned}
$$

which are substituted into (2.8) to yield the radial equation for the particle on the equatorial plane

$$
\frac{1}{2} E^{2}=\frac{1}{2} u^{r} u^{r}+V(r, E, L)
$$

with the effective potential

$$
V=\frac{\left(r-r_{+}\right)\left(r-r_{-}\right)}{2\left(r^{2}+l^{2}\right)} \kappa+\frac{\left(r-r_{+}\right)\left(r-r_{-}\right)}{2\left[\left(r^{2}+l^{2}\right)^{2}+4 l^{2}\left(r-r_{+}\right)\left(r-r_{-}\right)\right]}(L+2 l E)^{2} .
$$

For the null geodesics with $\kappa=0$, the effective potential $V(x, y)$ for the particles with the total energy per unit rest mass $E=0.1$ and angular momentum per unit rest mass $L=0.2 M$ is shown in the first graph in Fig. 1 where $x$ and $y$ denote the dimensionless variables $x=r / M$ and $y=l / M$, respectively. We observe here that there exist many peaks on the potential surface. In the third graph, we plot the surface $z=\left(x^{2}+y^{2}\right)^{2}+4 y^{2}\left(x^{2}-2 x-y^{2}\right)$ and the plane $z=0$, which shows the intersection curve corresponding to the vanishing values of the denominator of the second term in (2.11). Along this curve we have peaks of the potential surface in the first graph. In the second graph in Fig. 1, $V(x, y)$ is shown for the timelike geodesics of the particles with $E=0.1$ and $L=0.2 M$. In this case there exist peaks similar to those in the null geodesics. The potential surface is deformed due to the first term proportional to $\kappa$ in (2.11), which however does not affect the peak curve in the third graph since the denominator of this term is positive definite.

The fundamental equations of relativistic fluid dynamics can be obtained from the conservation of particle number and energy-momentum fluxes. In order to derive an equation for the conservation of particle numbers one can use the particle flux four vector $n u^{a}[20]$

$$
\nabla_{a}\left(n u^{a}\right)=\frac{1}{\sqrt{-g}} \nabla_{a}\left(\sqrt{-g} n u^{a}\right)=0
$$

where $n$ is the proper number density of particles measured in the rest frame of the fluid and $\nabla_{a}$ is the covariant derivative in the Taub-NUT curved manifold and $g=\operatorname{det} g_{a b}$. For steady state axisymmetric flow, the conservation of energy-momentum fluxes is similarly described by the Einstein equation [21]

$$
\nabla_{b} T_{a}^{b}=\frac{1}{\sqrt{-g}} \nabla_{b}\left(\sqrt{-g} T_{a}^{b}\right)=0,
$$

where the stress-energy tensor $T^{a b}$ for perfect fluid is given by

$$
T^{a b}=\rho u^{a} u^{b}+\left(g^{a b}+u^{a} u^{b}\right) P
$$

with $\rho$ and $P$ being the proper internal energy density, including the rest mass energy, and the isotropic gas pressure, respectively. The Einstein equation (2.13) can be rewritten in another covariant form

$$
u_{a} \nabla_{b}\left((P+\rho) u^{b}\right)+(P+\rho) u^{b} \nabla_{b} u_{a}+\nabla_{a} P=0 .
$$

On the other hand, from (2.1), (2.2) and (2.3) we can find the inverse of the Taub-NUT metric

$$
\begin{aligned}
g^{t t} & =-\frac{r^{2}+l^{2}}{\left(r-r_{+}\right)\left(r-r_{-}\right)}+\frac{4 l^{2}\left(r^{2}+l^{2}\right)}{\left[\left(r^{2}+l^{2}\right)^{2}+4 l^{2}\left(r-r_{+}\right)\left(r-r_{-}\right)\right] \sin ^{2} \theta}, \quad g^{r r}=\frac{\left(r-r_{+}\right)\left(r-r_{-}\right)}{r^{2}+l^{2}}, \quad g^{\theta \theta}=\frac{1}{r^{2}+l^{2}}, \\
g^{\phi \phi} & =\frac{r^{2}+l^{2}}{\left[\left(r^{2}+l^{2}\right)^{2}+4 l^{2}\left(r-r_{+}\right)\left(r-r_{-}\right)\right] \sin ^{2} \theta}, \quad g^{t \phi}=-\frac{2 l\left(r^{2}+l^{2}\right)}{\left[\left(r^{2}+l^{2}\right)^{2}+4 l^{2}\left(r-r_{+}\right)\left(r-r_{-}\right)\right] \sin ^{2} \theta} .
\end{aligned}
$$

Exploiting the inverse 4-metric $g^{a b}$ in (2.16) and acting the projection operator $g_{a b}+u_{a} u_{b}$ on the equation (2.15) we can obtain the general relativistic Euler equation on the direction perpendicular to the four velocity

$$
(P+\rho) u^{b} \nabla_{b} u_{a}+\left(g_{a b}+u_{a} u_{b}\right) \nabla^{b} P=0 .
$$


After some algebra, from (2.17) we obtain the radial component of the Euler equation for the steady state axisymmetric fluid

$$
\begin{aligned}
& \frac{d}{d r}\left(u^{r} u^{r}\right)+\frac{2 r-r_{+}-r_{-}}{r^{2}+l^{2}}-\frac{2\left(r-r_{+}\right)\left(r-r_{-}\right) r}{\left(r^{2}+l^{2}\right)^{2}}+\frac{4 l^{2} B}{\left(r^{2}+l^{2}\right) A} \\
& +\frac{4 l^{2} B}{\left(r-r_{+}\right)\left(r-r_{-}\right) A} u^{r} u^{r}+\frac{2}{P+\rho}\left(u^{r} u^{r}+\frac{\left(r-r_{+}\right)\left(r-r_{-}\right)}{r^{2}+l^{2}}\right) \frac{d P}{d r}=0
\end{aligned}
$$

where

$$
\begin{aligned}
& A=\left(r^{2}+l^{2}\right)^{2} \sin ^{2} \theta-4 l^{2}\left(r-r_{+}\right)\left(r-r_{-}\right) \cos ^{2} \theta \\
& B=\frac{\left(r^{2}+l^{2}\right)\left(r-r_{+}\right)\left(r-r_{-}\right)\left[\left(2 r-r_{+}-r_{-}\right)\left(r^{2}+l^{2}\right)-4 r\left(r-r_{+}\right)\left(r-r_{-}\right)\right]}{\left(r^{2}+l^{2}\right)^{2}+4 l^{2}\left(r-r_{+}\right)\left(r-r_{-}\right)} .
\end{aligned}
$$

In the vanishing $l$ limit, the result (2.18) is reduced to that of the (rotaing) Schwarzschild black hole [10].

Multiplying (2.15) by $u^{a}$ we can project it on the direction of the four velocity to obtain

$$
n u^{a}\left(\nabla_{a}\left(\frac{P+\rho}{n}\right)-\frac{1}{n} \nabla_{a} P\right)=0,
$$

where the continuity equation (2.12) has been used. The radial component of (2.20) yields

$$
\frac{d \rho}{d r}-\frac{P+\rho}{n} \frac{d n}{d r}=\frac{\Lambda-\Gamma}{u^{r}} .
$$

Here the energy loss $\Lambda$ and the energy gain $\Gamma$ are introduced to set the decrease in the entropy of inflowing gas equal to the difference $\Lambda-\Gamma$. Here it is interesting to see that the result (2.21) holds also in the (rotating) Schwarzschild black hole [10].

\section{GLOBAL EMBEDDINGS OF TAUB-NUT SPACETIME}

After tedious algebra, for the Taub-NUT spacetime in the region $r \geq r_{+}$we can obtain the $(6+5)$ global embedding Minkowski space (GEMS) structure

$$
d s^{2}=-\left(d z^{0}\right)^{2}+\left(d z^{1}\right)^{2}-\left(d z^{2}\right)^{2}+\left(d z^{3}\right)^{2}+\left(d z^{4}\right)^{2}+\left(d z^{5}\right)^{2}+\left(d z^{6}\right)^{2}-\left(d z^{7}\right)^{2}-\left(d z^{8}\right)^{2}+\left(d z^{9}\right)^{2}-\left(d z^{10}\right)^{2}
$$

with the coordinate transformations

$$
\begin{aligned}
& z^{0}=\kappa^{-1}\left(\frac{\left(r-r_{+}\right)\left(r-r_{-}\right)}{r^{2}+l^{2}}\right)^{1 / 2} \cos \frac{\theta}{2} \sinh \kappa(t+2 l \phi), \\
& z^{1}=\kappa^{-1}\left(\frac{\left(r-r_{+}\right)\left(r-r_{-}\right)}{r^{2}+l^{2}}\right)^{1 / 2} \cos \frac{\theta}{2} \cosh \kappa(t+2 l \phi), \\
& z^{2}=\kappa^{-1}\left(\frac{\left(r-r_{+}\right)\left(r-r_{-}\right)}{r^{2}+l^{2}}\right)^{1 / 2} \sin \frac{\theta}{2} \sinh \kappa(t-2 l \phi), \\
& z^{3}=\kappa^{-1}\left(\frac{\left(r-r_{+}\right)\left(r-r_{-}\right)}{r^{2}+l^{2}}\right)^{1 / 2} \sin \frac{\theta}{2} \cosh \kappa(t-2 l \phi), \\
& z^{4}=\left(\frac{\left(r^{2}+l^{2}\right)^{2}+4 l^{2}\left(r-r_{+}\right)\left(r-r_{-}\right)}{r^{2}+l^{2}}\right)^{1 / 2} \sin \theta \cos \phi \\
& z^{5}=\left(\frac{\left(r^{2}+l^{2}\right)^{2}+4 l^{2}\left(r-r_{+}\right)\left(r-r_{-}\right)}{r^{2}+l^{2}}\right)^{1 / 2} \sin \theta \sin \phi, \\
& z^{6}=\left(\frac{\left(r^{2}+l^{2}\right)^{2}+4 l^{2}\left(r-r_{+}\right)\left(r-r_{-}\right)}{r^{2}+l^{2}}\right)^{1 / 2} \cos \theta, \\
& z^{7}=\left(4 l^{2}+\frac{1}{4} \kappa^{-2}\right)^{1 / 2}\left(\frac{\left(r-r_{+}\right)\left(r-r_{-}\right)}{r^{2}+l^{2}}\right)^{1 / 2} \cos \theta
\end{aligned}
$$




$$
\begin{aligned}
z^{8} & =\left(4 l^{2}+\frac{1}{4} \kappa^{-2}\right)^{1 / 2}\left(\frac{\left(r-r_{+}\right)\left(r-r_{-}\right)}{r^{2}+l^{2}}\right)^{1 / 2} \sin \theta, \\
z^{9}= & \int d r \frac{\left\{p(r)\left[\left(r_{+}-r_{-}\right)\left(r^{2}+l^{2}\right)^{2}+\left(r_{+}^{2}+l^{2}\right) s(r)\right]+q(r)\left(r_{+}^{2}+l^{2}\right)\left(r_{+}+r_{-}\right) l^{2}\right\}^{1 / 2}}{\left(r-r_{-}\right)^{1 / 2}\left(r_{+}-r_{-}\right)\left(r^{2}+l^{2}\right)^{3 / 2}} \equiv f(r), \\
z^{10}= & \int d r\left(\frac{p(r)\left(r_{+}^{2}+l^{2}\right)\left(r_{+}+r_{-}\right) l^{2}+q(r)\left[\left(r_{+}-r_{-}\right)\left(r^{2}+l^{2}\right)^{2}+\left(r_{+}^{2}+l^{2}\right) s(r)\right]}{\left(r-r_{-}\right)\left(r_{+}-r_{-}\right)^{2}\left(r^{2}+l^{2}\right)^{3}}\right. \\
\left.+\frac{\left[r\left(r^{2}+l^{2}\right)^{2}+2 l^{2}\left(2 r-r_{+}-r_{-}\right)\left(r^{2}+l^{2}\right)-4 l^{2} r\left(r-r_{+}\right)\left(r-r_{-}\right)\right]^{2}}{\left[\left(r^{2}+l^{2}\right)^{2}+4 l^{2}\left(r-r_{+}\right)\left(r-r_{-}\right)\right]\left(r^{2}+l^{2}\right)^{3}}\right)^{1 / 2} & \equiv g(r),
\end{aligned}
$$

where $\kappa$ is given by

$$
\kappa^{-2}=\frac{16}{3}\left[\left(\frac{r_{+}^{2}+l^{2}}{r_{+}-r_{-}}\right)^{2}+l^{2}\right],
$$

and the positive definite functions are given by

$$
\begin{aligned}
& p(r)=\left(r_{+}-r_{-}\right) r^{3}+r_{+}\left(r_{+}-r_{-}\right) r^{2}+l^{2} r_{+} r_{-}, \\
& q(r)=\left(2 r_{+}^{2}+3 l^{2}\right) r_{-} r+l^{2}\left(r_{+}^{2}+r_{+} r_{-}+2 l^{2}\right), \\
& s(r)=\left(r_{+}-r_{-}\right) r^{2}+2\left(l^{2}-r_{+} r_{-}\right) r .
\end{aligned}
$$

Here note that $\kappa$ in (3.3) is not the surface gravity, different from those in the embeddings in the static black holes without the shift functions [13, 16, 19, 22].

Next, in order to investigate the region $r \leq r_{+}$we rewrite the Taub-NUT 4-metric as

$$
d s^{2}=\left(-\frac{\Delta}{\Sigma}\right)(d t+2 l \cos \theta d \phi)^{2}-\left(-\frac{\Sigma}{\Delta}\right) d r^{2}+\Sigma\left(d \theta^{2}+\sin ^{2} \theta d \phi^{2}\right)
$$

in terms of the positive definite lapse function inside the event horizon $r_{+}$

$$
-\frac{\Delta}{\Sigma}=\frac{\left(r_{+}-r\right)\left(r-r_{-}\right)}{r^{2}+l^{2}}
$$

to yield the (6+5) GEMS structure (3.1) with the coordinate transformation

$$
\begin{aligned}
z^{0} & =\kappa^{-1}\left(\frac{\left(r_{+}-r\right)\left(r-r_{-}\right)}{r^{2}+l^{2}}\right)^{1 / 2} \cos \frac{\theta}{2} \cosh \kappa(t+2 l \phi), \\
z^{1} & =\kappa^{-1}\left(\frac{\left(r_{+}-r\right)\left(r-r_{-}\right)}{r^{2}+l^{2}}\right)^{1 / 2} \cos \frac{\theta}{2} \sinh \kappa(t+2 l \phi), \\
z^{2} & =\kappa^{-1}\left(\frac{\left(r_{+}-r\right)\left(r-r_{-}\right)}{r^{2}+l^{2}}\right)^{1 / 2} \sin \frac{\theta}{2} \cosh \kappa(t-2 l \phi), \\
z^{3} & =\kappa^{-1}\left(\frac{\left(r_{+}-r\right)\left(r-r_{-}\right)}{r^{2}+l^{2}}\right)^{1 / 2} \sin \frac{\theta}{2} \sinh \kappa(t-2 l \phi), \\
z^{7} & =\left(4 l^{2}+\frac{1}{4} \kappa^{-2}\right)^{1 / 2}\left(\frac{\left(r_{+}-r\right)\left(r-r_{-}\right)}{r^{2}+l^{2}}\right)^{1 / 2} \cos \theta, \\
z^{8} & =\left(4 l^{2}+\frac{1}{4} \kappa^{-2}\right)^{1 / 2}\left(\frac{\left(r_{+}-r\right)\left(r-r_{-}\right)}{r^{2}+l^{2}}\right)^{1 / 2} \sin \theta, \\
z^{9} & =f(r), \\
z^{10} & =g(r),
\end{aligned}
$$

where $\left(z^{4}, z^{5}, z^{6}\right), f(r)$ and $g(r)$ are the same as those in (3.2). 


\section{CONCLUSIONS}

In conclusion, we studied the Taub-NUT spacetime to investigate hydrodynamic properties of the perfect fluid whirling inward toward the Taub-NUT spacetime along a conical surface. On the Taub-NUT manifolds we then constructed the $(6+5)$ dimensional global embeddings inside and outside the event horizons of the manifold. We also studied the effective potentials of particles on the equatorial planes of the Taub-NUT spacetime in terms of the gravitational magnetic monopole strength of the source, total energy and angular momentum per unit rest mass of the particle.

\section{Acknowledgments}

The work of STH was supported by the Korea Research Foundation Grant funded by the Korean Government (MOEHRD) (KRF-2006-331-C00071). SWK was supported by the Korea Research Foundation Grant funded by the Korean Government (MOEHRD) (KRF-2006-312-C00498).

[1] A. Taub, Ann. Math. 53, 472 (1951); E.T. Newman, L. Tamburino, and T. Unti, J. Math. Phys. 4, 915 (1963).

[2] S.W. Hawking, Phys. Lett. A 60, 81 (1977).

[3] N.S. Manton, Phys. Lett. B 110, 54 (1985).

[4] M.F. Atiyah and N. Hitchin, Phys. Lett. A 107, 21 (1985).

[5] S. Hewson, Class. Quantum Grav. 13, 1739 (1996).

[6] T.C. Kraan and P. van Baal, Phys. Lett. B 428, 268 (1998).

[7] B. Carter, Comm. Math. Phys. 10, 280 (1968).

[8] D. Bini, C. Cherubini, M. de Mattia and R.T. Jantzen, Gen. Rel. Grav. 35, 2249 (2003); D. Bini, C. Cherubini, R.T. Jantzen and B. Mashhoon, Class. Quantum Grav. 20, 457 (2003); D. Bini, C. Cherubini, R.T. Jantzen and B. Mashhoon, Phys. Rev. D 67, 084013 (2003).

[9] Z.W. Chong, G.W. Gibbons, H. Lü and C.N. Pope, Phys. Lett. B 609, 124 (2005).

[10] S.T. Hong and S.W. Kim, gr-qc/0503079.

[11] K. Schwarzschild, Sitzber. Deut. Akad. Wiss. Berlin, KI. Math.-Phys. Tech. pp. 189-196 (1916).

[12] M. Spivak, Differential Geometry (Publish or Perish, Berkeley 1975) Vol 5, Chapter 11.

[13] S. Deser and O. Levin, Class. Quant. Grav. 14, L163 (1997); S. Deser and O. Levin, Class. Quant. Grav. 15, L85 (1998); S. Deser and O. Levin, Phys. Rev. D 59, 064004 (1999).

[14] S.W. Hawking, Comm. Math. Phys. 42, 199 (1975); J.D. Bekenstein, Phys. Rev. D 7, 2333 (1973); R.M. Wald, Quantum Field Theory in Curved Spacetime and Black Hole Thermodynamics (The University of Chicago Press, Chicago 1994); J.D. Brown, J. Creighton and R.B. Mann, Phys. Rev. D 50, 6394 (1994); R.M. Wald, Living Rev. Rel. 4, 6 (2001).

[15] W.G. Unruh, Phys. Rev. D 14, 870 (1976); P.C.W. Davies, J. Phys. A 8, 609 (1975).

[16] S.T. Hong, Gen. Rel. Grav. 36, 1919 (2004).

[17] H.Z. Chen, Y. Tian and Y.H. Gao, JHEP 0410, 011 (2004); H.Z. Chen and Y. Tian, gr-qc/0410077.

[18] M. Banados, C. Teitelboim, and J. Zanelli, Phys. Rev. Lett. 69, 1849 (1992); M. Banados, M. Henneaux, C. Teitelboim, and J. Zanelli, Phys. Rev. D 48, 1506 (1993).

[19] S.T. Hong, Phys. Lett. B 578, 187 (2004).

[20] L.D. Landau and E.M. Lifschitz, Fluid Mechanics (Pergamon, Oxford 1977).

[21] S.L. Shapiro, Ap. J. 189, 343 (1974).

[22] S.T. Hong, Y.W. Kim and Y.J. Park, Phys. Rev. D 62, 024024 (2000); S.T. Hong, W.T. Kim, Y.W. Kim and Y.J. Park, Phys. Rev. D 62, 064021 (2000); S.T. Hong, W.T. Kim, J.J. Oh and Y.J. Park, Phys. Rev. D 63, 127502 (2001). 